\title{
News on two jets in Lupus $3^{\star}$
}

\author{
F. Comerón ${ }^{1}$ and M. Fernández ${ }^{2}$ \\ ${ }^{1}$ ESO, Karl-Schwarzschild-Strasse 2, 85748 Garching bei München, Germany \\ e-mail: fcomeron@eso.org \\ 2 Instituto de Astrofísica de Andalucía, CSIC, Glorieta de la Astronomía 3, 18008 Granada, Spain \\ e-mail: matilde@iaa.es
}

Received 23 October 2010 / Accepted 24 January 2011

\begin{abstract}
Context. Jets from solar-type and low-mass stars are typical manifestations of stellar youth. Shocks along these jets produce visible, generally fast-moving Herbig-Haro objects whose proper motions are easily measured in nearby star-forming regions using images taken just a few years apart. Herbig-Haro objects have now been observed in association with objects close to the substellar boundary. Aims. We present second-epoch observations of the central area of the Lupus 3 star-forming region that include two of its most interesting Herbig-Haro systems. One is HH 228, produced by the classical T Tauri star Th 28 (=Sz 102), whereas the other is HH 600, which has its origin in the very low-mass star Par-Lup3-4.

Methods. Narrow-band imaging through filters centered on the $\mathrm{H} \alpha$ and the [SII] lines was obtained with the FORS2 instrument at the Very Large Telescope (VLT) in mid-2010. The images obtained were compared to others obtained in early 2003, leading to the discovery of HH 600.

Results. New Herbig-Haro objects are found at large distances from Th 28 and to be associated with it, representing an extension of the $\mathrm{HH} 228$ jet to a projected distance of $0.32 \mathrm{pc}$ from Th 28. The farthest Herbig-Haro object is HH 989, whose possible relationship with Th 28 had been already suggested in a previous study but is now kinematically confirmed. We find other likely Herbig-Haro objects whose proper motions are less indicative of a connection with Th 28 , but which may be caused by oblique shocks near the outer walls of its jet. For the HH 600 jet, the knot discovered by ourselves in 2003 to the southeast of Par-Lup3-4 is found to have clearly moved and faded. Using high resolution spectroscopy obtained in 2003 and the proper motion that we can measure now, we determine a spatial velocity of $170 \pm 30 \mathrm{~km} \mathrm{~s}^{-1}$. The northwestern jet is found to have grown in prominence in the intervening years. The possible relationship of other Herbig-Haro objects in the region with Th 28, Par-Lup3-4, and other young stellar objects in the area is discussed.
\end{abstract}

Key words. stars: formation - Herbig-Haro objects

\section{Introduction}

Collimated jets are an ubiquitous feature of the earliest stages of star formation. They are produced in the inner regions of the environment of low- and intermediate-mass stars, resulting from the interaction between the magnetic fields of a newly formed young stellar object and its surrounding disk and envelope (Hartmann 1998; Bally et al. 2007; Shang et al. 2007; Ray et al. 2007; Pudritz et al. 2007). Time variations and sudden changes in the ejection velocities of the jets, instabilities in their launching region, and interactions with the surrounding interstellar medium give rise to shocks that are visible as the compact, fast-moving nebula represented by Herbig-Haro (HH) objects. $\mathrm{HH}$ objects thus provide a record of the past history of an outflow, over timescales that can be in some instances as long as the entire duration of the protostellar stage of its central source (Bally 2009). In the nearest star-forming regions, their high spatial velocities translate into large proper motions easy to measure in only a few years, thus providing an accessible tool to study the evolution of the mechanical properties of the jets and the timing of past events. Proper motions provide in addition a simple way

* Based on observations collected with the Very Large Telescope (VLT) at the European Southern Observatory, Paranal, Chile, under observing programme 385.C-0535(A); and on VLT archive observations originally obtained under programme 71.C-0429(D). to confirm the nature of $\mathrm{HH}$ objects and to unambiguously link them to their central sources, which may not be straightforward in regions densely populated by young stellar objects.

Near the center of the T Tauri association Lupus 3 are two remarkable young stellar objects driving spatially resolved outflows. The T Tauri star Th 28 (Thé 1962), also known as Sz 102 (Schwartz 1977) or Krautter's star, is the source of the fast bipolar jet HH 228 (Krautter 1986; Graham \& Heyer 1988). Another object in the same region, Par-Lup3-4 (Comerón et al. 2003), is a very low-mass star close to the substellar limit that was found by Fernández \& Comerón (2005) to have a faint associated jet, HH 600 (Comerón \& Reipurth 2006), which is one of the lowest mass objects known to drive a spatially resolved jet.

In this paper, we report the discovery of new, faint $\mathrm{HH}$ objects to the east of Th 28 that happen to be closely aligned with the axis defined by the known knots of HH 228. Proper motions are measured for all of them, as well as for HH 989, first identified by Wang \& Henning (2009) and whose relationship to Th 28 we are able to confirm kinematically. The distance of the newly identified knots related to Th 28 extends the size of the detectable jet to $\sim 0.3 \mathrm{pc}$ from the central star, and provides a fossil record of its outflow activity over the past thousand years. We also present second-epoch observations of the $\mathrm{HH} 600$ jet that allow us to obtain the first measurement of its proper motion. This 
complements the observations of Fernández \& Comerón (2005), allowing us to determine the spatial velocity of the jet, and confirms its orientation close to the plane of the sky.

\section{Observations and measurements}

The new observations reported here were obtained on the nights of 15/16 April, 5/6 May, and 6/7 May 2010 using the FORS2 camera and spectrograph at the VLT in imaging mode. Two narrow-band filters, covering respectively the $\mathrm{H} \alpha$ line at $6563 \AA$ and the [SII] lines at $6716 \AA$ and $6731 \AA$, were used to obtain relatively deep images of a small region of the Lupus 3 cloud roughly centered on Par-Lup3-4. The images were obtained with the high-resolution collimator of FORS2 yielding a scale of $0^{\prime \prime} 126$ pixel $^{-1}$ on the detector, which we used in the standard $2 \times 2$ binned mode. The field covered by each individual frame is $4^{\prime} 3 \times 4^{\prime} 3$, with vignetting toward the corners caused by the collimator optics. The observations in each filter consisted of three separate pointings, each with an exposure time of $1043 \mathrm{~s}$, with telescope offsets in-between. Owing to the combination of pointing geometry and corner vignetting, the coadded frame in each filter has an irregular contour, the longest dimensions in the east-west and north-south directions being 5'0 across.

The area covered by these observations nicely overlaps with our previous images obtained with the FORS1 instrument at the VLT and very similar narrow-band filters in February 2003, reported in Fernández \& Comerón (2005). The reader is referred to that paper for a description of the FORS1 observations. The 7.2 years elapsed between both sets of observations, and the similar sensitivity reached in both, allow us to obtain the first measurement of the proper motion of HH 600. They also allow us to search for possible faint, previously unidentified $\mathrm{HH}$ objects in the region that should be easily noticeable because of their clear displacement between both epochs, caused by their large spatial velocities. The area covered by both sets of observations includes much of the region east of Th 28 , and is thus almost ideally suited to searching for further extensions of the HH 228 system of $\mathrm{HH}$ objects beyond the knots that have been identified thus far.

The February 2003 images were astrometrically calibrated using the cataloged positions of 2MASS stars in the field as a reference. The images obtained in 2010 were then distortioncorrected to match the 2003 images and resampled by means of the GEOMAP and GEOTRANS tasks on IRAF, this time taking as reference all the common, non-saturated stars detected above the $5 \sigma$ level between the 2003 and 2010 images. The quality of the distortion correction varies across the field, as the most obscured areas are rather devoid of stars and only a coarse reference grid can be established for them.

Proper motions for the new $\mathrm{HH}$ objects identified in this work to be associated with Th 28 , as well as the proper motion of HH 453 (see Sect. 3), were measured with respect to their nearest stars in the images, which are in all cases unrelated to the Lupus clouds. The proper motions of background stars in the direction of Lupus are centered very near the position $\left(\mu_{\alpha} \cos \delta\right.$, $\left.\mu_{\delta}\right)=(0,0)$ (Mugrauer \& Neuhäuser 2005), where $\mu_{\alpha} \cos \delta$ and $\mu_{\delta}$ are the proper motions in right ascension and declination, respectively. Therefore, no correction was made to the proper motions of the $\mathrm{HH}$ objects measured with respect to their reference stars. For HH 600, the proper motion of the jet knot was determined relative to its central source, Par-Lup3-4.

Given the extension of the objects, their irregular shapes, the noise in the images, and perhaps the slightly evolving intrinsic morphology, it was found that careful visual inspection leading to the identification of specific, easily recognizable features within each object turned out to be the most reliable way of choosing a reference point for the measurement of the proper motions. This could be done with a varying degree of accuracy depending on the morphology and brightness of each object. The uncertainty in the location of this reference point is the dominant source of uncertainty, except in the case of HH 453 (see Sect. 3) where the uncertainty due to the scarcity of nearby reference stars is comparable.

\section{Results}

\subsection{Th 28 and the $\mathrm{HH} 228 / \mathrm{HH} 989$ jet}

The system of HH objects associated with Th 28 is oriented close to the east-west direction and appears to be moving in a direction nearly perpendicular to the line of sight, as inferred by comparing the large proper motions (Krautter 1986) to their radial velocities (Graham \& Heyer 1988). This is consistent with the faintness of the central source, which is probably due to the obscuration by a circumstellar disk seen nearly edge-on (Hughes et al. 1994), despite the lack of the pronounced dip in the mid-infrared spectral energy distribution near $8 \mu \mathrm{m}$ (Chapman et al. 2007) that is characteristic of a near edge-on viewing geometry (D'Alessio et al. 1999). The basis of the jet has been studied in depth thanks to the high spatial resolution observations provided by STIS at the Hubble Space Telescope, which have allowed a detailed analysis of the physical conditions near the launching region both along and across the jet, as well as the identification of hints of rotation (Coffey et al. 2004, 2007) and an estimate of the mass-loss rate in the outflow, which is found to be a remarkably high $\sim 1.2 \times 10^{-8} M_{\odot} \mathrm{yr}^{-1}$ (Coffey et al. 2008). Further probing of the innermost region of the jet using nearinfrared hydrogen and [FeII] lines was recently presented by the same authors (Coffey et al. 2010). The different outflow and inflow components in the immediate circumstellar environments of Th 28 were discussed by Comerón \& Fernández (2010) based on spatially unresolved high resolution spectroscopy of a variety of emission lines. An accretion rate of $\sim 6.3 \times 10^{-8} M_{\odot} \mathrm{yr}^{-1}$ is inferred from these observations, which, with the caveat of the uncertainties in both estimates, suggests a high outflow-to-inflow mass ratio. The system of knots composing HH 228 is also discussed in that paper, as well as by Wang \& Henning (2009), who provide a complete census of $\mathrm{HH}$ objects known thus far in the Lupus 3 region.

The observations discussed here map most of the eastern jet of Th 28. This component of the jet has a significant negative radial velocity, measured at -62 and $-82 \mathrm{~km} \mathrm{~s}^{-1}$ at the positions of $\mathrm{HH} 228 \mathrm{E}_{1}$ and $\mathrm{HH} 228 \mathrm{E}_{2}$ respectively (Graham \& Heyer 1988). Our comparison between the $\mathrm{H} \alpha$ images obtained in 2003 and 2010 reveals five previously unidentified faint nebulae in the proximities of Th 28 or the axis of its jet that show significant or suspected displacement between both epochs. The new objects are HH 228 E, HH 228 E, HH 453, HH 989 B, and $\mathrm{HH} 989 \mathrm{C}$. The somewhat confusing nomenclature is meant to preserve the denominations already assigned to the previously known objects HH $228 \mathrm{E}_{1}, \mathrm{HH} 228 \mathrm{E}_{2}$ and $\mathrm{HH} 989$, and to reflect the likely association and proximity of the new objects to them. $\mathrm{HH} 453$ is most likely unrelated to the Th 28 jet, thus has been assigned a different, unused $\mathrm{HH}$ catalog number. The location of these nebulae, as well as the already known HH 989, is shown in Fig. 1. The new $\mathrm{HH}$ objects are fainter and more extended than those closer to Th 28, thus making it difficult to determine their proper motion to the same level of accuracy attainable for 


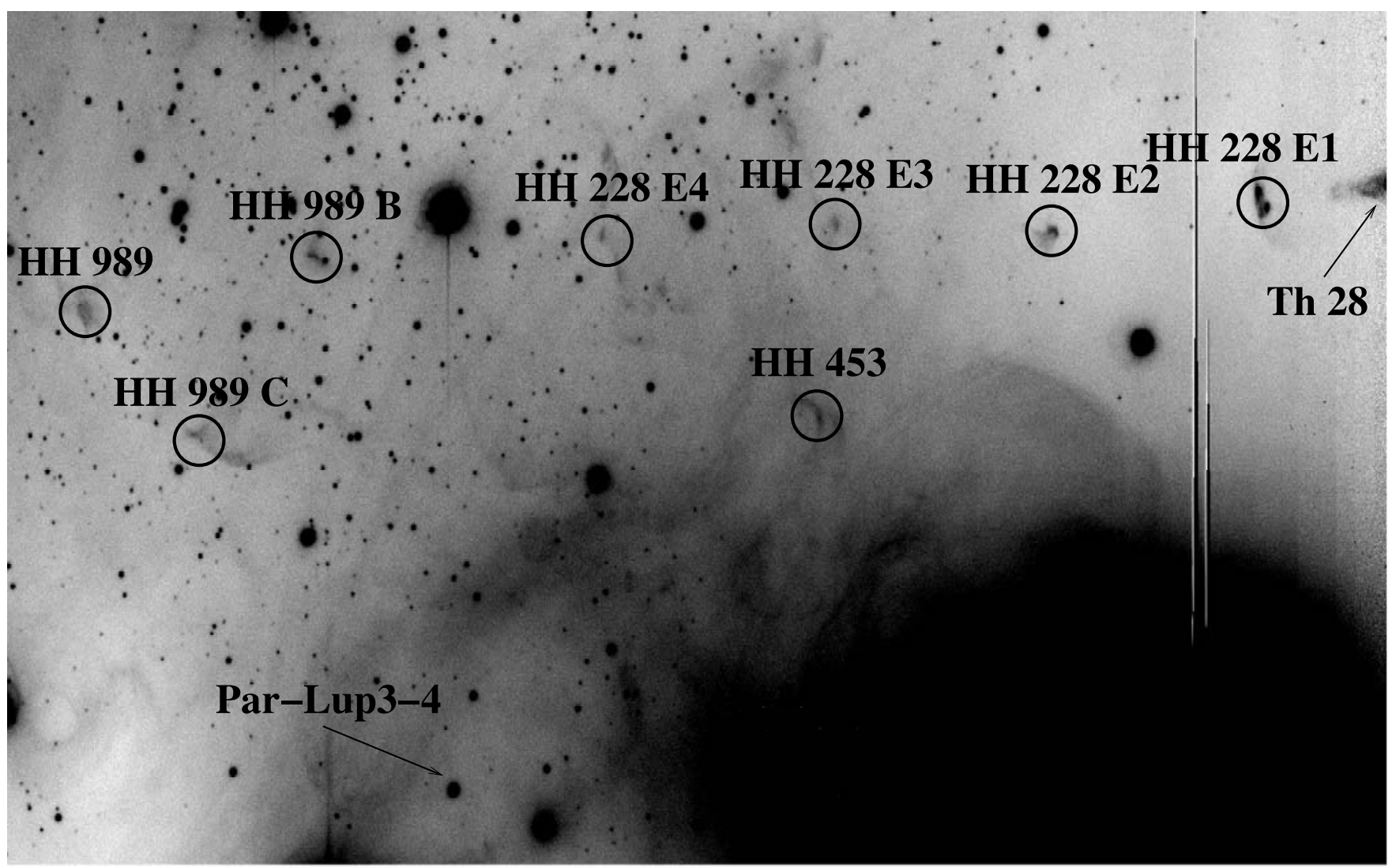

Fig. 1. A general $\mathrm{H} \alpha$ view of the field covered by our observations, obtained from our FORS1 observations in 2003. The objects discussed in the present study are marked. Th 28 is just outside the right edge of the frame, but its inner jet and the knots composing HH 228 can be identified. The field covers an area of $6^{\prime} 1 \times 3^{\prime} 3$, with north to the top and east to the left.

Table 1. Positions, relative proper motions and velocities of knots associated with Th 28 .

\begin{tabular}{|c|c|c|c|c|c|c|c|}
\hline Object & $\begin{array}{l}\alpha(2000) \\
\quad \text { (Epoch }\end{array}$ & (Epoch 2010.4) & \multicolumn{2}{|c|}{$\left(\operatorname{arcsec}_{\mathrm{yr}^{-1}}\right)$} & $\begin{array}{c}\lambda \\
(\operatorname{arcsec})\end{array}$ & $\begin{array}{c}v \\
\left(\mathrm{~km} \mathrm{~s}^{-1}\right)\end{array}$ & Reference point \\
\hline HH $228 \mathrm{E}_{3}$ & $16: 08: 42.4$ & $-39: 03: 30$ & $+0.118 \pm 0.05$ & $+0.294 \pm 0.05$ & 147 & 300 & center of the nebula \\
\hline HH $228 \mathrm{E}_{4}$ & 160848.3 & $-39: 03: 23$ & $+0.371 \pm 0.03$ & $+0.066 \pm 0.05$ & 210 & 357 & peak of the central condensation \\
\hline HН 989 В & $16: 08: 54.7$ & $-39: 03: 28$ & $+0.135 \pm 0.07$ & $-0.022 \pm 0.03$ & 287 & 130 & center of the condensation \\
\hline HH 989 C & $16: 08: 57.7$ & $-39: 04: 17$ & $-0.058 \pm 0.05$ & $-0.092 \pm 0.03$ & 322 & 103 & peak of the eastern condensation \\
\hline HН 989 & 16:09:00.1 & $-39: 03: 41$ & $+0.328 \pm 0.05$ & $+0.049 \pm 0.05$ & 347 & 314 & approximate center of the condensation \\
\hline
\end{tabular}

the previously known $\mathrm{HH}$ objects, due to both the compactness and the longer time baseline over which the latter have been measured. However, the 7.2 yr baseline available for the new objects provides a sound basis to assess their possible relationship to known young stellar objects in the region, particularly to HH 228.

A close-up view of each of these knots in $\mathrm{H} \alpha$ is presented in Fig. 2. The large proper motions of most of them are already apparent upon visual inspection. Table 1 shows the positions, proper motions relative to Th $28\left(\Delta \mu_{\alpha} \cos \delta, \Delta \mu_{\delta}\right)$, angular distances to Th 28 projected along the axis of its jet $(\lambda)$, and velocity on the plane of the sky with respect to Th 28 $(v)$. The adopted proper motion for Th 28 is taken from the UCAC3 catalog (Zacharias et al. 2010) as $\left(\mu_{\alpha} \cos \delta, \mu_{\delta}\right)=$ $\left(+0^{\prime \prime} 0046 \mathrm{yr}^{-1},-0^{\prime \prime} 0310 \mathrm{yr}^{-1}\right)$, which differs somewhat from the bulk proper motion of the Lupus clouds derived by Makarov (2007) of $\left(\mu_{\alpha} \cos \delta, \mu_{\delta}\right)=\left(-0^{\prime \prime} 017 \mathrm{yr}^{-1},-0^{\prime \prime} 027 \mathrm{yr}^{-1}\right)$. Data for $\mathrm{HH} 453$ are presented separately in Table 2. With the single exception of $\mathrm{HH} 989$, the knots are detected in $\mathrm{H} \alpha$ alone, being undetected in the [SII] images.
Table 2. Position and proper motion of HH 453.

\begin{tabular}{ll}
\hline \hline$\alpha(2000)$ & $16: 08: 43.0$ \\
$\delta(2000)$ & $-39: 04: 05$ \\
$\mu_{\alpha} \cos \delta\left(\operatorname{arcsec} \mathrm{yr}^{-1}\right)$ & $-0.088 \pm 0.07$ \\
$\mu_{\delta}\left(\operatorname{arcsec} \mathrm{yr}^{-1}\right)$ & $+0.035 \pm 0.07$ \\
Reference point & peak intensity at the arc feature \\
\hline
\end{tabular}

\subsection{Par-Lup3-4 and HH 600}

The faint object Par-Lup3-4 was first identified by Comerón et al. (2003) thanks to its outstanding emission-line spectrum, superimposed on a faint continuum corresponding to a M5 spectral type. Par-Lup3-4 is surrounded by large amounts of circumstellar material, which make it one of the brightest young stellar objects in the Lupus 3 region at mid-infrared wavelengths (Merín et al. 2008). An analysis of its visible-to-mid infrared spectral energy distribution shows that it can be well modeled by a very low-mass star surrounded by a thick flaring disk seen nearly edge-on, thus explaining the unusually low luminosity of 

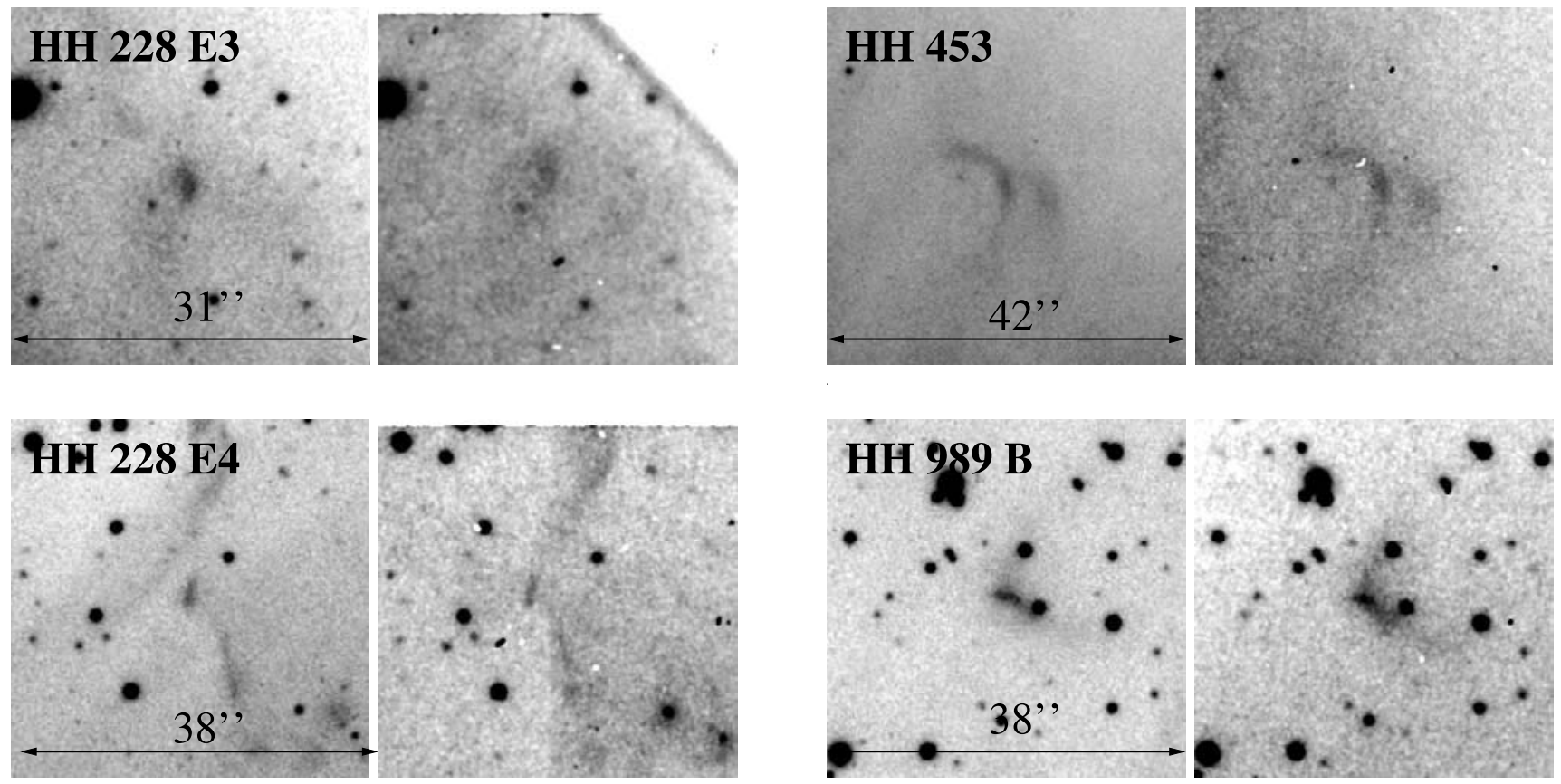

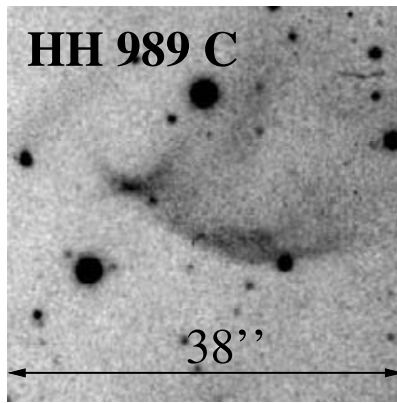

2003

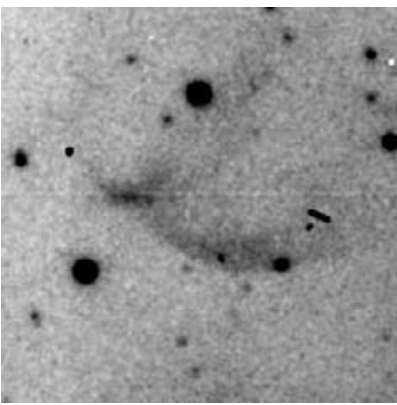

2010

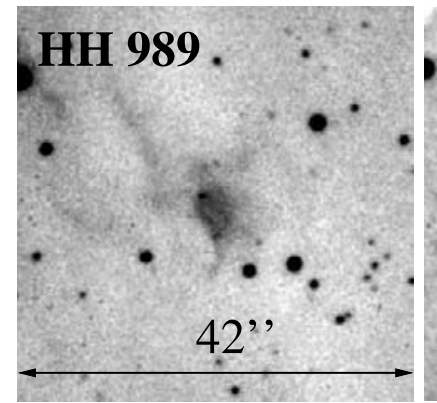

2003

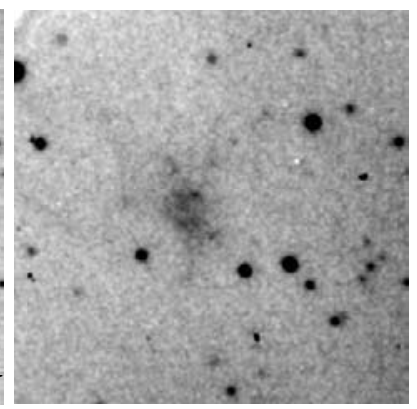

2010

Fig. 2. $\mathrm{H} \alpha$ images obtained in 2003 and 2010 of the five moving knots in the proximities of the Th 28 jet, plus a possibly moving arc-shaped nebula southeast of Th 28 . The displacement in the intervening 7.2 years images can be noticed for most of them by comparison with stars in the field. North is up and East to the left. Note the different scales of the images.

the central object at visible and near-infrared wavelengths, in which it is mostly seen in scattered light (Huélamo et al. 2010). A short bipolar jet, $\mathrm{HH}$ 600, was found by Fernández \& Comerón (2005), who presented high-resolution spectroscopy indicating velocities of only $20.1 \mathrm{~km} \mathrm{~s}^{-1}$ along the line of sight.

The new observations of Par-Lup3-4 show that there have been important changes in its system of jets, as seen in Fig. 3. In the 2003 observations, reported in Fernández \& Comerón (2005), the most outstanding feature was a knot located 1 " 3 from the central star. The knot could be clearly seen in the [SII] image and could be seen in the $\mathrm{H} \alpha$ image as well. A faint extension of the jet pointing southeast was visible beyond the knot, as well as traces of a counterjet toward the northwest. In the image of 2010, the appearance of both jets is much more symmetric with both the southeast and the northwest jet displaying similar intensities. The southeast jet ends in a distinct condensation at 2" 55 from Par-Lup3-4, which most likely is the knot seen in the 2003 images much closer to the star, thus allowing us to easily measure the jet proper motion relative to Par-Lup3-4. Interestingly, all this structure is revealed by the $[\mathrm{SII}]$ image only, and no trace of the jet down to the level of sensitivity of our observations is seen
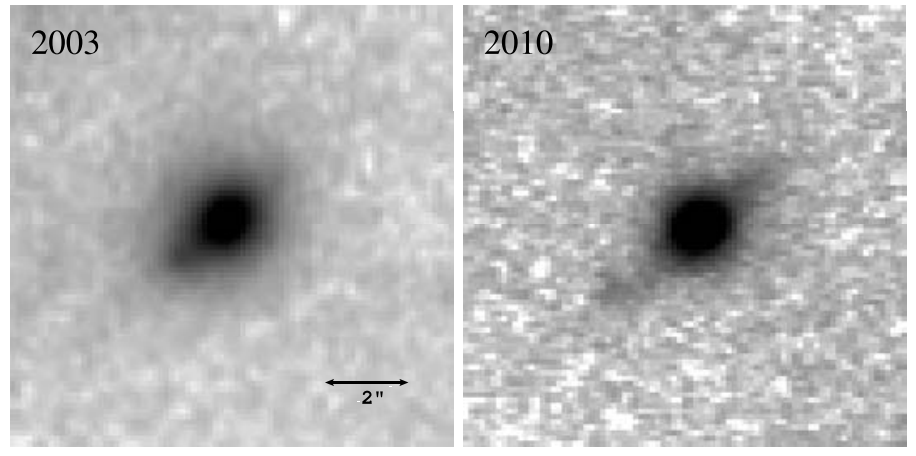

Fig. 3. [SII] images of Par-Lup3-4 and its faint jet. The grey scale is logarithmic in both images to enhance the structure of the jet. North is up and east to the left.

in the $\mathrm{H} \alpha$ images. This is in sharp contrast to most of the knots belonging to the Th 28 jet or in its proximities where, as noted above, the opposite situation happens. 


\section{Discussion}

\subsection{The distant extensions of the $\mathrm{HH} 228$ jet}

The positions and proper motions of two of the objects listed in Table 1, HH $228 \mathrm{E}_{4}$ and $\mathrm{HH} 989$, clearly indicate their association with HH 228 and extend the known length of the jet. Their location is very close to the axis defined by the HH $228 \mathrm{E}_{1}$ and $E_{2}$ knots (Graham \& Heyer 1988), and their proper motions point directly away from Th 28 . HH $228 \mathrm{E}_{4}$ is moving through a region that projects it against possibly unrelated nebulosity, as can be inferred from Fig. 2 by noting that an upper ridge of emission running approximately north-south remains fixed in the images, whereas the bright, compact patch near the center of the image and the nebulosity south of it are rapidly proceeding eastward. HH 989 is composed of a compact nebulosity preceded by nebulous wisps toward the east, which also share its motion across space. The nature of HH 989 as the extension of HH 228 was already suggested by Wang \& Henning (2009), and is now unambiguously confirmed by ourselves. HH 989 is the only knot that we detect in both $\mathrm{H} \alpha$ and [SII] images. The peak intensity of $\mathrm{HH} 228 \mathrm{E}_{4}$ in $\mathrm{H} \alpha$ is similar to that of $\mathrm{HH} 989$, but its nondetection in our [SII] images, as well as in those used by Wang \& Henning for their study, indicates that the excitation of HH 989 is higher than that of knot $\mathrm{HH} 228 \mathrm{E}_{4}$ and possibly the other knots.

Assuming a distance of $200 \mathrm{pc}$ to the Sun, the tangential velocities of $\mathrm{HH} 228 \mathrm{E}_{4}$ and $\mathrm{HH} 989$ are very high at $357 \pm$ $30 \mathrm{~km} \mathrm{~s}^{-1}$ and $314 \pm 50 \mathrm{~km} \mathrm{~s}^{-1}$, respectively. The relative proper motions are, however, below those measured for $\mathrm{HH} 228 \mathrm{E}_{1}$ and HH $228 E_{2}$, for which Fernández \& Comerón (2005) find, respectively, $0.43 \pm 0.03 \operatorname{arcsec}_{\mathrm{yr}}{ }^{-1}$ and $0.47 \pm 0.03 \operatorname{arcsec}_{\mathrm{yr}} \mathrm{r}^{-1}$. Assuming that the jet knots have been moving away from Th 28 at a constant velocity since the outflow events that produced them, we infer that these events took place approximately 95, 207, 558, and 1042 years ago for $\mathrm{HH} 228 \mathrm{E}_{1}, \mathrm{HH} 228 \mathrm{E}_{2}$, $\mathrm{HH} 228 \mathrm{E}_{4}$, and HH 989, respectively. Taken at face value, this might indicate that the pace of outflow events triggering the formation of knots has increased in recent centuries. However, it is also possible that knots resulting from old outflow events that occurred in-between have faded away and are no longer recognizable.

HH 989 represents the most distant extension of the Th 28 eastern jet discovered thus far, which has a length of at least $0.32 \mathrm{pc}$. Given that it lies near the edge of the field covered by our observations, the presence of detectable $\mathrm{HH}$ objects located even further away cannot be discarded, although none was identified by Wang \& Henning (2009), whose images cover regions further east of the one examined by ourselves. Those authors speculate that a bright and more distant object, HH 78 (Reipurth \& Graham 1988), which lies near but not exactly on the axis of the Th 28 jet, may represent an additional extension of this structure. We conclude, however, that this is extremely unlikely. Deep infrared observations of the region (Nakajima et al. 2003) show that the visible portion of $\mathrm{HH} 78$ can be traced as a well collimated jet up to the precise position of the Class 0 object Lupus 3 MMS (Tachihara et al. 2007), which is almost surely at the origin of this jet. The near-infrared part of the jet is bright in the $K_{\mathrm{S}}$ band, probably because of shock-excited $\mathrm{H}_{2}$ emission coincident with the molecular outflow detected by Tachihara et al. (2007). The relative arrangement in the sky of the infrared and visible portions suggests that the jet originates at a location deeply embedded in the cloud hosting Lupus 3 MMS and moves toward the side of the cloud facing in our direction, revealing itself as the visible counterpart of $\mathrm{HH} 78$ as it emerges.
$\mathrm{HH} 228 \mathrm{E}_{3}$ and $\mathrm{HH} 989 \mathrm{C}$ also have large proper motions, but not as extreme as those of the four HH knots just discussed. They are both offset from the axis and moving away from the jet. Their positional/kinematical association with Th 28 is thus not as straightforward as that of $\mathrm{HH} 228 \mathrm{E}_{1}, \mathrm{E}_{2}, \mathrm{E}_{4}$, and $\mathrm{HH} 989$. Nevertheless, the formation of these slower outwardly moving, off-axis knots is a feature reproduced by hydrodynamical simulations of pulsed jets. They appear as the bow shock produced by an ejection event that moves in the wake of a previously produced knot interacts with material near and drives an oblique shock into the walls of the jet (see for instance Fig. 8 of Bonito et al. 2010). The plausibility of this scenario according to numerical simulations, and the absence of any viable candidates to be the driving engines of $\mathrm{HH} 228 \mathrm{E}_{3}$ and $\mathrm{HH} 989 \mathrm{C}$, encourages us to accept this explanation of the location and motion of those two objects.

The nature of HH 989 B is somewhat more uncertain. It appears as a bright patch very close to the axis of the jet, but its proper motion is small. Our proper motion measurement takes as reference point the center of the brightest condensation, which is elongated in the east-west direction, the same as the presumed direction of motion. However, given the different depths of the images from 2003 and 2010 and perhaps the morphological evolution of the nebula over this period, it is possible that the proper motion that we derive may be the spurious effect of either noise in the images or a physical change of shape of the knot. If the reported proper motion is confirmed by future observations spanning a longer time, it is likely that $\mathrm{HH} 989 \mathrm{~B}$ has then a similar origin as that discussed above for $\mathrm{HH} 228 \mathrm{E}_{3}$ and $\mathrm{HH} 989 \mathrm{C}$. However, the possibility that it is an unrelated object, and perhaps not even a $\mathrm{HH}$ object, remains open.

The fairly regular distribution of the string of knots, and the timescales on the order of a century involved between the production of consecutive knots, suggests that the episodic activity may be due to a close eccentric binary (Reipurth 2000), in which the knots are produced by bursts repeated on an orbital timescale around the time of the periastron passage of the companion. Although no evidence of binarity has been found in Th 28, future high spatial resolution observations may provide support for this scenario.

Finally, HH 453 is almost certainly unrelated to Th 28 , and is listed here only for completeness. Its proper motion is uncertain, partly owing to the scarcity of reference stars in that part of the region, which may lead to an inaccurate correction for differential field distortion between the 2003 and 2010 images. Its westward proper motion is consistent with the arc shape of the knot, which is reminiscent of a bow shock. Very similar morphologies are observed in other HH objects such as HH 61, HH 63, Re 6, or HH 77 (Reipurth \& Graham 1988).

The source of HH 453 is uncertain. The apsis of the bow shock points backwards toward the proximities of $\mathrm{Sz} \mathrm{109,} \mathrm{a} \mathrm{very}$ low mass star with spectral type M6.5 (Comerón et al. 2003) located 60" away. However, in contrast to the case with the most $\mathrm{HH}$-driving sources, Sz 109 has a very modest emission-line spectrum, where the only emission line is $\mathrm{H} \alpha$ with an equivalent width of only $15 \AA$. Another intriguing possibility is that HH 453 might actually be associated with Par-Lup3-4, as discussed in Sect. 4.2.

\subsection{The evolution of the $\mathrm{HH} 600$ jet}

Being close to the borderline between very low-mass stars and brown dwarfs, Par-Lup3-4 is an important object for the study 
of the properties of jets produced in that range of masses. Increasing evidence is being found of jets driven by very lowmass stars (Stecklum \& Meusinger 2007) and brown dwarfs (Whelan et al. 2009). In the latter case, the evidence of jets is mostly found in the close proximity of the central object and can be resolved only by high-resolution spectroastrometric techniques, although evidence of a spatially well-resolved molecular outflow driven by a brown dwarf was presented by Phan-Bao et al. (2008). Par-Lup3-4 thus provides a case in which an optical jet from a very low-mass star can be both spatially and kinematically resolved, and where its evolution with time on short timescales can be followed in detail.

The clear displacement of the knot in HH 600 in the intervening 7.2 years allows us to easily determine its velocity in the plane of the sky relative to Par-Lup3-4, which amounts to $\left(\Delta \mu_{\alpha} \cos \delta, \Delta \mu_{\delta}\right)=(+0.136 \pm 0.015,-0.107 \pm 0.015) \operatorname{arcsec}^{-1} r^{-1}$, translating into a velocity of $(168 \pm 30) \mathrm{km} \mathrm{s}^{-1}$ in the plane of the sky at the adopted distance of $200 \mathrm{pc}$. This velocity is in the typical range for classical T Tauri stars, and above those of the brown dwarf jets investigated by Whelan et al. (2009), although the latter compose a statistically small sample. In addition to the radial velocity of $20.1 \pm 1.5 \mathrm{~km} \mathrm{~s}^{-1}$ determined by Fernández \& Comerón (2005), we obtain a spatial velocity of $170 \mathrm{~km} \mathrm{~s}^{-1}$ and an inclination of the jet with respect to the plane of the sky of only $6^{\circ} 7 \pm 1^{\circ} 4$. This confirms that the as yet unresolved disk of Par-Lup3-4 is indeed very close to edge-on, as already hinted by Fernández \& Comerón (2005) and independently supported by Huélamo et al. (2010). The ejection event that caused the knot is thus estimated to have happened in early 1995.

Although the northwestern jet of $\mathrm{HH} 600$ has brightened since 2003, the knot in the southeastern jet has faded significantly, and we estimate its [SII] flux to have dropped to $30 \%$ of the brightness recorded in 2003. Fernández \& Comerón (2005) noted that the knot could be seen in the $2003 \mathrm{H} \alpha$ images, which is not the case in the 2010 images. If the observed knot is a typical product of the pulses of Par-Lup3-4, we conclude that those structures fade away too quickly to be observed at angular distances from Par-Lup3-4 longer than a few arcseconds. Interestingly, the opposite behavior between both epochs is observed for Par-Lup3-4 itself, as its flux in both the [SII] and $\mathrm{H} \alpha$ passbands was higher in 2010 by $\sim 30-40 \%$. This increase could be due to enhanced emission lines in 2010 with respect to 2003, perhaps indicative of a higher level of outflow activity. The increase could also be due to variability in the continuum level, which is known to happen according to the broad-band imaging of Comerón et al. (2003). Unfortunately, no broad-band observations are available in 2010 allowing us to decide between both possibilities. In either case, continued monitoring of Par-Lup3-4 might soon reveal the birth of another jet knot.

The quick fading of the knot in the HH 600 jet appears to conflict with the suggestion by Wang \& Henning (2009) that two distant HH objects discovered by them, HH 900 and HH 991, might originate in Par-Lup3-4. Their suggestion is based on the location of those two objects along the extension of the ParLup3-4 jet only, and unfortunately we are unable to verify this interpretation by measuring the kinematics as both objects are outside the field of our 2010 observations. We note that an alternative, and perhaps likelier source for HH 990 is Lupus 3 MMS, already mentioned in Sect. 4.1, as HH 990 is also in the extension of the HH 78 jet. The origin of HH 991, located at 177" from Par-Lup3-4, remains however uncertain. On the other hand, it is intriguing that $\mathrm{HH} 453$, discussed in Sect. 4.1, also lies approximately along the extension of the HH 600 northwestern jet, although at a shorter distance (131") than HH 991. The limited precision of our kinematic measurements cannot exclude that HH 453 might be moving radially away from Par-Lup3-4. Were future observations to confirm the association of some of these three objects with Par-Lup3-4, it would be evidence of a far more active outflow ejection history having taken place several centuries ago.

\section{Summary}

New narrow-band [SII] and $\mathrm{H} \alpha$ imaging has been presented of a field containing the eastern jet of Th 28 and the very low-mass young stellar object Par-Lup3-4. A comparison with observations obtained with similar instrumentation over seven years earlier leads to the following conclusions:

- The eastern jet powered by Th 28 , which contains the Herbig-Haro objects $\mathrm{HH} 228 \mathrm{E}_{1}$ and $\mathrm{E}_{2}$, is found to extend out to a distance of at least $0.3 \mathrm{pc}$ from the central source. The most distant part of the jet identified thus far is HH 989, whose association with Th 28 and $\mathrm{HH} 228$ we are able to confirm kinematically.

- Four additional HH objects are identified near the axis defined by $\mathrm{Th} 28$ and $\mathrm{HH}$ 989, which we denominate HH 228 E $_{3}$, HH 228 E, HH 989 B, and HH 989 C. Proper motions are measured for these objects. The proper motion of $\mathrm{HH} 228 \mathrm{E}_{4}$ is very large and similar to those of $\mathrm{HH} 228 \mathrm{E}_{1}, \mathrm{E}_{2}$, and $\mathrm{HH} 989$, confirming its association with the jet. HH $228 \mathrm{E}_{3}$, HH $989 \mathrm{~B}$ and $\mathrm{HH} 989 \mathrm{C}$ have measurable proper motions that differ from those of $\mathrm{HH} 228 \mathrm{E}_{1}$, $\mathrm{E}_{2}, \mathrm{E}_{4}$, and $\mathrm{HH} 989$, but given their proximity to the axis of the jet and the direction of motion we suggest that they are caused by oblique shocks near the walls of the jet. Unlike $\mathrm{HH} 228 \mathrm{E}_{1}, \mathrm{E}_{2}$, and $\mathrm{HH}$ 989, the new HH objects are detected only in $\mathrm{H} \alpha$ and not in [SII]. The known and new $\mathrm{HH}$ objects trace variability in the jet powered by Th 28 over the last millenium, and hint to major variability events on the timescales of one century.

- The small bipolar jet powered by the very low-mass star ParLup3-4, HH 600, is also detected in our new observations. A compact knot in the jet, detected 1" 3 from the central star in both $\mathrm{H} \alpha$ and [SII] images obtained in 2003, is found to have moved to $2^{\prime \prime} 55$ after 7.2 years, implying a velocity in the plane of the sky of $168 \pm 30 \mathrm{~km} \mathrm{~s}^{-1}$ and a direction of motion nearly perpendicular to the line of sight when the proper motion measurement is combined with the radial velocity obtained from observations in 2003. The knot is not detected in $\mathrm{H} \alpha$ in the new images, and has faded significantly in [SII]. At the same time, the opposite side of the jet has gained prominence in the intervening years, whereas Par-Lup3-4 itself is $30-40 \%$ brighter in the 2010 observations than in the 2003 ones.

- A likely, arc-shaped new HH object, HH 453, is detected in the proximities of $\mathrm{HH} 228$. It is almost certainly unrelated to Th 28 , but its powering source remains uncertain. On the basis of its position and shape, it might be associated with Par-Lup3-4, like another likely HH object, HH 991, located at a comparable distance on the opposite side of Par-Lup34. However, the actual association of both HH objects with Par-Lup3-4 still awaits confirmation.

The results presented in this paper underscore the importance of deep imaging and monitoring in revealing the evolution of the jets associated with young stellar objects over widely different timescales, ranging from millenia to a few years. They also 
encourage further observations of the two systems studied here to explore both possible major outbursts in the distant past, and near-real-time follow-up of new ejection events in the coming years.

Acknowledgements. It is a pleasure to thank the Paranal Science Operations staff for the execution of the observations in Service Mode, and to Bodo Ziegler at the ESO's User Support Department, within the ESO's Data Management and Operations Division, for the support with the Phase 2 preparation of these observations. We are thankful to Bo Reipurth for his advice on the naming of the new Herbig-Haro objects reported here, and for the assignment of catalog numbers. Comments by an anonymous referee leading to significant improvements in the content and presentation of this paper are gratefully acknowledged. M.F. acknowledges financial support both from the Spanish Ministry of Science and Innovation (MICINN) through grant AYA2007-64052, and from the Regional Ministry of Education and Science of the Regional Government of Andalusia through grants TIC-101 and TIC-4075.

\section{References}

Bally, J. 2009, in Protostellar Jets in Context, ed. K. Tsinganos, T. Ray, \& M. Stute, Astroph. Sp. Sci. Proc. Ser. (Springer Verlag)

Bally, J., Reipurth, B., \& Davis, C. J. 2007, in Protostars and Planets V, ed. B. Reipurth, D. Jewitt, \& K. Keil (University of Arizona Press)

Bonito, R., Orlando, S., Peres, G., et al. 2010, A\&A, 511, A42

Chapman, N. L., Lai, S.-P., Mundy, L. G., et al. 2007, ApJ, 667, 288

Coffey, D., Bacciotti, F., Woitas, J., Ray, T. P., \& Eislöffel, J. 2004, ApJ, 604, 758

Coffey, D., Bacciotti, F., Ray, T. P., Eislöffel, J., \& Woitas, J. 2007, ApJ, 663, 350

Coffey, D., Bacciotti, F., \& Podio, L. 2008, ApJ, 689, 1112

Coffey, D., Bacciotti, F., Podio, L., \& Nisini, B. 2010, ApJ, 719, 505

Comerón, F. 2008, The Lupus clouds, in Handbook of Star Forming Regions, 2, ed. B. Reipurth, ASP Monographs
Comerón, F., \& Reipurth, B. 2006, A\&A, 458, L21

Comerón, F., \& Fernández, M. 2010, A\&A, 511, A10

Comerón, F., Fernández, M., Baraffe, I., Neuhäuser, R., \& Kaas, A. A. 2003, A\&A, 406, 1001

D’Alessio, P., Calvet, N., Hartmann, L., Lizano, S., \& Cantó, J. 1999, ApJ, 527, 893

Fernández, M., \& Comerón, F. 2005, A\&A, 440, 1119

Graham, J. A., \& Heyer, M. H. 1988, PASP, 100, 1529

Hartmann, L. 1998, Accretion Processes in Star Formation (Cambridge Univ. Press)

Huélamo, N., Bouy, H., Pinte, C., et al. 2010, A\&A, 523, A42

Hughes, J. H., Hartigan, P., Krautter, J, \& Kelemen, J. 1994, AJ, 108, 1071

Krautter, J. 1986, A\&A, 161, 195

Makarov, V. V. 2007, ApJ, 658, 480

Merín, B., Jørgensen, J., Spezzi, L., et al. 2008, ApJS, 177, 551

Mugrauer, M., \& Neuhäuser, R. 2005, AN, 326, 701

Nakajima, Y., Nagata, T., Sato, S., et al. 2003, AJ, 125, 1407

Phan-Bao, N., Riaz, B., Lee, C.-P., et al. 2008, ApJ, 689, L141

Pudritz, R. E., Ouyed, R., Fendt, Ch., \& Brandenburg, A., 2007, in Protostars and Planets V, ed. B. Reipurth, D. Jewitt, \& K. Keil (University of Arizona Press)

Ray, T., Dougados, C., Bacciotti, F., Eislöffel, J., \& Chrysostomou, A. 2007, in Protostars and Planets V, ed. B. Reipurth, D. Jewitt, \& K. Keil (Univ. Arizona Press)

Reipurth, B. 2000, AJ, 120, 3177

Reipurth, B., \& Graham, J. A. 1988, A\&A, 202, 219

Schwartz, R. D. 1977, ApJS, 35, 161

Shang, H., Li, Z.-Y., \& Hirano, N. 2007, in Protostars and Planets V, ed. B. Reipurth, D. Jewitt, \& K. Keil (Univ. Arizona Press)

Stecklum, B., \& Meusinger, H. 2007, Ap\&SS, 311, 63

Tachihara, K., Rengel, M., Nakajima, Y., et al. 2007, ApJ, 659, 1382

Thé, P. S. 1962, Contrib. Bosscha Obs., 15

Wang, H., \& Henning, Th. 2009, AJ, 138, 1072

Whelan, E. T., Ray, T. P., Podio, L., Bacciotti, F., \& Randich, S. 2009, ApJ, 706, 1054

Zacharias, N., Finch, C., Girard, T., et al. 2010, AJ, 139, 2184 